\title{
Ovarian neoplastic changes in dairy cows with adenomyosis - histopathological study
}

\author{
Maria Katkiewicz \\ 45 Ebro Str., 01- 490 Warsaw, Poland \\ m.katkiewicz@gmail.com \\ Received: December 29, 2019 Accepted: July 27, 2020
}

\begin{abstract}
Introduction: This paper presents the results of the microscopic examination of dairy cow ovaries. Material and Methods: The examined dairy cows were culled in a slaughterhouse. In all of them, pathological changes $(\mathrm{n}=82)$ associated with adenomyosis had been previously diagnosed, and additionally in some cows $(n=18)$ so had mammary gland cell injury, including mastitis purulenta. Results: There was vast variability among the investigated individuals in the degree of disease and type of pathological changes in the examined tissue specimens. In all examined ovaries, the most prominent lesions were neoplastic metaplasia of various cell types. They were PEComa, rete ovarii cell neoplasm, granulosa cell tumour, and a single case of haemangioma cavernosum, and the first of these types of ovarian tumours was the prominent neoplasia. In some cases, they grew simultaneously with the other types of tumours, but tumour tissue never extended beyond the ovarian capsule. Sometimes, the ovarian tumours were of microscopic volume, for instance the foci of granulosa cell tumours. Conclusion: The lack of changes in ovarian anatomic structure, and minute neoplastic tissue foci, make it impossible to diagnose these lesions in the ovaries of living animals. The presented original data may be valuable in understanding the aetiology of dairy cow infertility, as well as in facilitating urgently needed research into findings new methods, which might be used in the diagnosis of neoplastic diseases ante mortem.
\end{abstract}

Keywords: dairy cow, ovary neoplasia.

\section{Introduction}

Granulosa cell tumours are the most frequent type of neoplastic changes described in cows' ovaries. The unknown types of ovarian tumors are presented in this study. They were found in the ovaries of slaughtered dairy cows and related to the uterine pathological changes characteristic of the adenomyosis disease process.

PEComa (periarterial epithelioid cell neoplasm) belongs to the group of mesenchymal tumours (4). This type of neoplasia was described in humans in various organs, including the ovaries and uterus $(2,13)$. Two decades ago PEComa appeared in the WHO classification of human neoplastic diseases (16). Recently, PEComa was found in dairy cows' ovaries (in press), as well as in uterine biopsy samples, the latter as the first case described in animal pathology (6).

A PEComa tumour develops as the result of the neoplastic transformation of the periarterial epithelioid cells. There are no published data concerning the factors, which may play a crucial role in the mechanism of this type of neoplastic cell transformation. The pathological changes in the other organs may suggest the role of a primary hormonal imbalance present in diseased animals only.

The second type of tumours found in the examined dairy cows' ovaries was neoplasia of rete ovarii cells. These tumours are also found in other animals $(9,12)$. The minute volume of the rete ovarii cell structure, as well as their neoplastic lesions, make it impossible to recognise them in living animals. However, the disturbances in of the physiological function of the ovary caused by these tumours is well known $(1,14)$. Rete ovarii epithelial cells function is regulated by ovarian hormones stimulation, through the pathway of specific receptors found in these cells (10). Therefore, it is evident that rete cells are susceptible to any hormonal imbalance, and such is proved by the appearance of pathological changes due to these disease factors $(5,7,8)$.

This article presents the various types of ovarian pathological changes found in dairy cows with 
documented uterine adenomyosis. Among them, the most prominent lesions are ovarian neoplasms.

\section{Material and Methods}

Both ovaries were taken from dairy cows $(n=100)$, of various ages, culled in a slaughterhouse. In all examined cows, uterine adenomyosis pathological changes were confirmed. Among these cows, a subgroup of animals $(n=18)$ was identified in which pathological udder cellular tissue changes, including mastitis purulenta inflammatory lesions, were microscopically diagnosed.

The examined tissue specimens were fixed in neutral $10 \%$ buffered formalin, embedded in paraffin, sectioned, and stained with haematoxylin and eosin (HE). The evaluation of the specimens was performed using a light microscope.

\section{Results}

Uterus histopathological changes. In all examined uterus specimens, pathological changes characteristic of the adenomyosis disease process were diagnosed. They were the proliferation of basal mucosal glands, and mucosa interstitial tissue, atrophy of glands of the superficial mucosa layer, and small foci of inflammatory cell infiltration in the mucosa layer (Figs 1, 2, 3). Among the examined cows, these types of pathological changes were found to highly varying degrees.

Ovaries. In the group of 82 dairy cows of which the udder tissue was not examined, a PEComa was found in 13 animals. The tumours were found in both ovaries in seven cows, and in two cows a PEComa was growing in a single ovary. In two cases there were PEComa and numerous small foci of granulosa cell tumours. PEComa was also associated with adenoma of the rete ovarii in two cases.

In the subgroup of 18 dairy cows with documented mammary glands pathological changes, PEComa tumours were found in all examined ovaries. PEComa were growing in both ovaries in 14 instances and only in one ovary in four. PEComa tumours were associated with foci of granulosa cell tumour in seven cases and with fibroadenoma of the rete ovarii in three ovaries. In one case, haemangioma cavernosum associated with PEComa and rete ovarii fibroadenoma was found.

The tumour cell structure had characteristic focal blood vessel proliferation. In the periphery of the blood vessels, the walls were cuffed with neoplastic epithelioid cells (Fig. 4). In less advanced tumour tissue growth, the proliferation was mainly localised in the ovarian hilus. In cases with more advanced tumour proliferation, PEComa tissue reached the ovarian cortex border, but never grew beyond the ovarian capsule (Figs 5 and 6). There was some relation observed between the stages of the tumour tissue proliferation and the structure of
PEComa neoplastic cells. In advanced tumour tissue growth, the neoplastic cells were smaller, with condensed hyperchromatic nuclei (Fig. 7), which may indicate the progress in their anaplastic changes.

Among all examined cows, great variability in the degree of neoplastic tissue infiltration into the gonadal stromal tissue was observed, and also was in the related degree of atrophy of the follicular ovarian cortex. There was also necrobiosis (Fig. 8) of the primary and secondary follicles. In the majority of examined ovaries, follicular cysts were observed (Fig. 9), and in single cases, luteal cysts were also found.

The second type of neoplasia was observed in rete ovarii cells. This type of neoplastic lesions was found in 36 adenomyotic dairy cows $(n=82)$. There were 27 rete ovarii fibroadenomas (Fig. 10) and nine rete ovarii adenocarcinomas (Fig. 11). In the subgroup of dairy cows with documented mammary glands diseases $(\mathrm{n}=18)$, the rete ovarii cell neoplastic lesions were noted in three cases accompanied by ovarian PEComa and small disseminated foci of granulosa cell tumours (Fig. 12). In an ovary with the prominent rete ovarii fibroadenoma $(n=1)$, there were small disseminated foci of PEComa - and in another, besides rete ovarii fibroadenoma, there were small foci of PEComa and granulosa cell tumours. In a third ovary, there were rete ovarii fibroadenoma cysticum, small foci of PEComa, and a vast field of haemangioma cavernosum. In this subgroup of dairy cows, rete ovarii fibroadenoma was found in both ovaries in four cases.

\section{Discussion}

The data presented in this research indicate that PEComa in dairy cows may develop frequently and is accompanied by adenomyosis, mammary glands lesions. The diagnosis of these ovarian tumours may not be performed in living animals, and this may explain why this type of tumours growth has been unknown up to now, in contrast for example, to the widely known granulosa cell tumours $(3,11)$. Probably, the same situation exists in the clinical diagnosis of human PEComa, and as a consequence, on the rare occasions, it is diagnosed that type of neoplastic growth is described as a very rare disease (15).

The results of the present research may suggest some coincidence of the aetiology of the epithelioid cell neoplastic metaplasia with the primary hormonal imbalance present in the examined cows. That hormonal imbalance was proved by the documented uterine and mammary gland cell pathological changes observed in the examined dairy cows.

A very clear relationship was found to exist between mammary gland cell pathology and the inflammatory process with ovarian PEComa. These findings may indicate that there are some interaction mechanisms present between the endocrine and the immune system functions in diseased animals. 


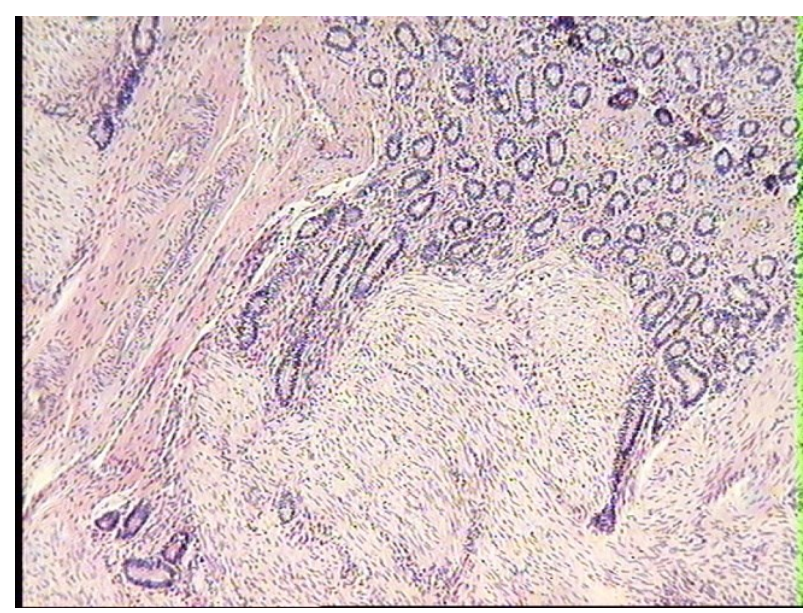

Fig. 1. Proliferation of mucosal basal glands into the muscular layer in the uterus of dairy cow. HE, $10 \times$

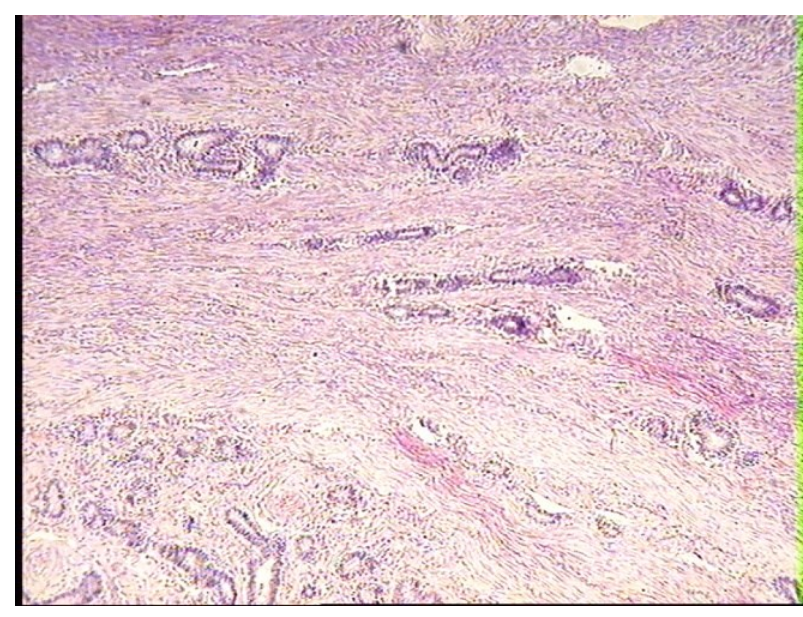

Fig. 2. Foci of the endometrial pathological tissue disseminated in the muscular layer in the uterus of dairy cow. HE, 10×

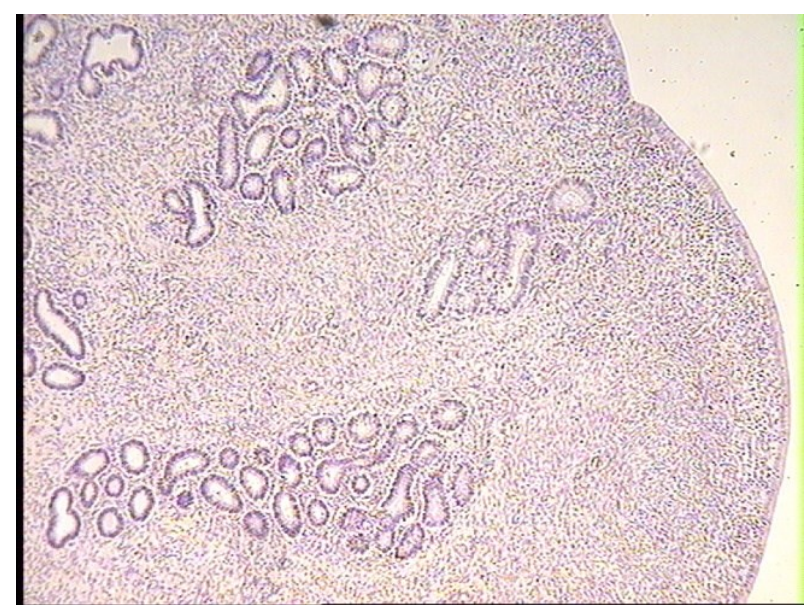

Fig. 3. Interstitial fibrosis of the superficial, functional mucosa layer, accompanied by glands atrophy in the uterus of dairy cow. HE, 10×

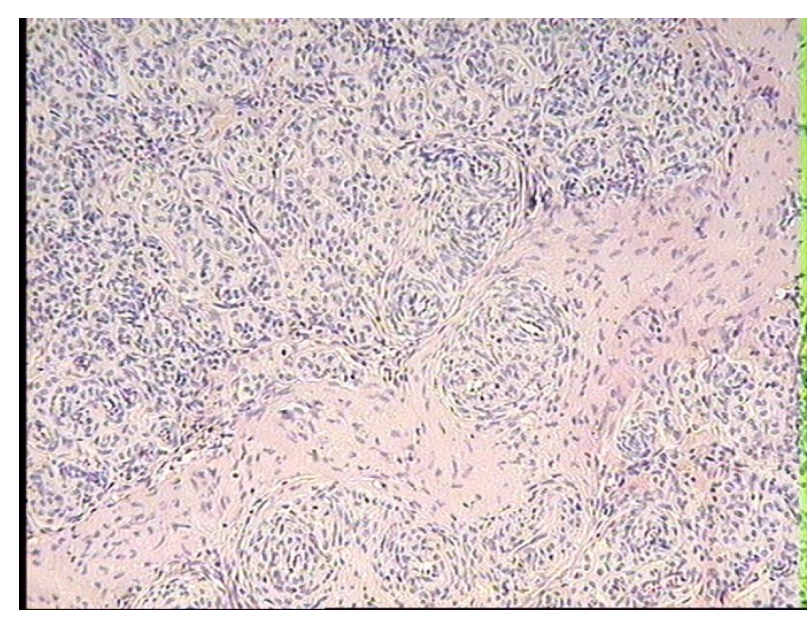

Fig. 4. Focus of PEComa neoplastic tissue in the ovary of a dairy cow with an adenomyotic uterus. HE, $20 \times$

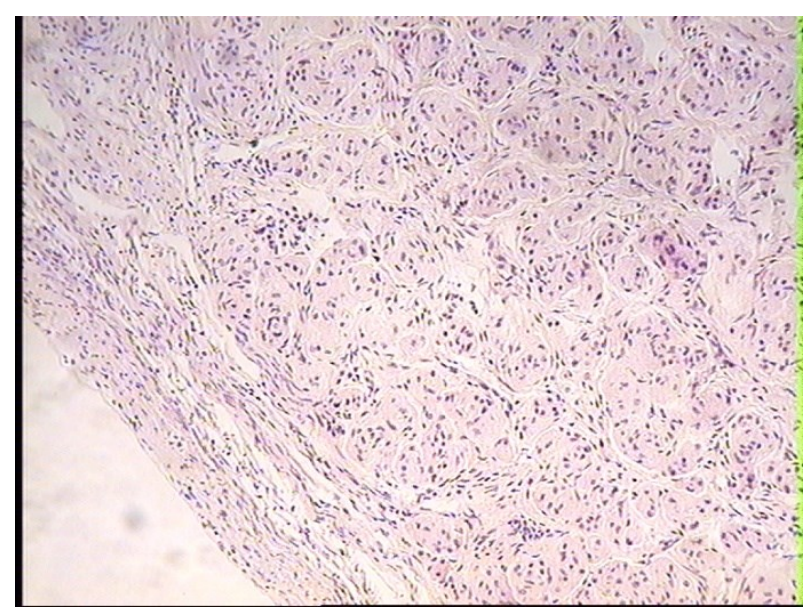

Fig. 5. PEComa tissue overgrowth to the ovarian capsule in the ovary of a dairy cow with an adenomyotic uterus. HE, 20×

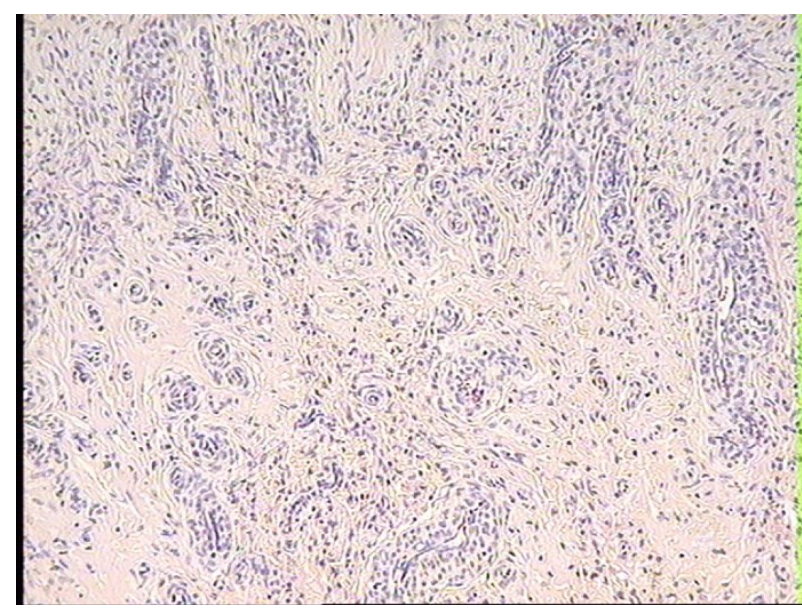

Fig. 6. Neoplastic PEComa tissue infiltrating ovarian stroma in the ovary of a dairy cow with an adenomyotic uterus. HE, 10× 


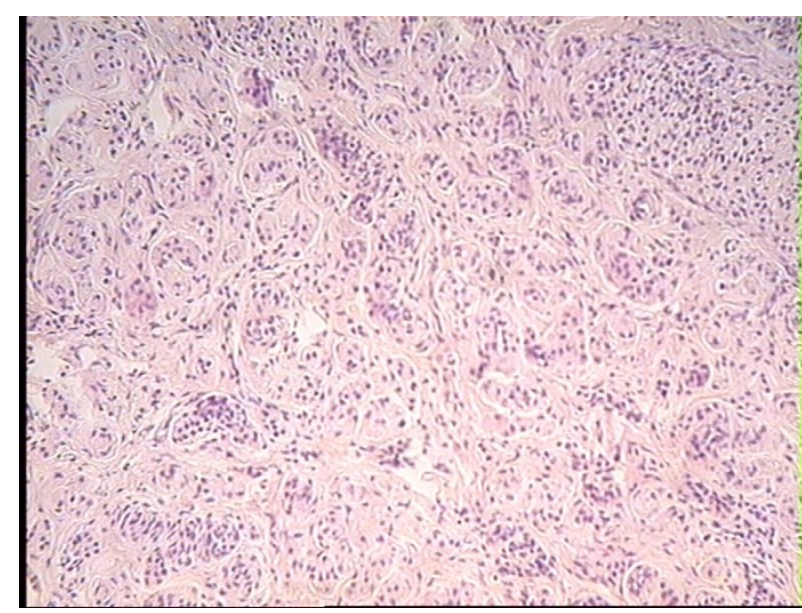

Fig. 7. Anaplasia of cells seen in the PEComa tissue infiltrating the gonadal stroma in the ovary of a dairy cow with an adenomyotic uterus. HE, 20×

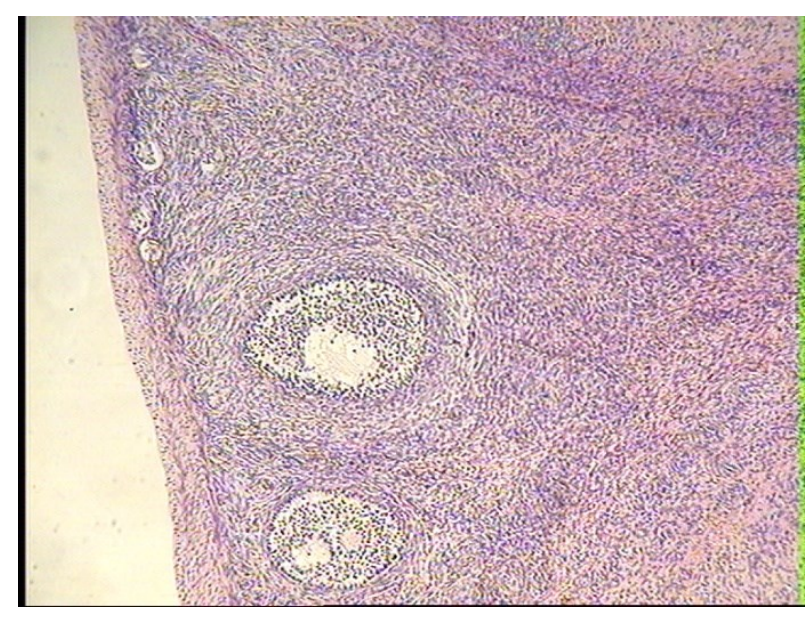

Fig. 8. PEComa tumour neoplasia and in the ovary and necrobiotic changes in primary ovarian follicles in a dairy cow. HE, $10 \mathrm{x}$

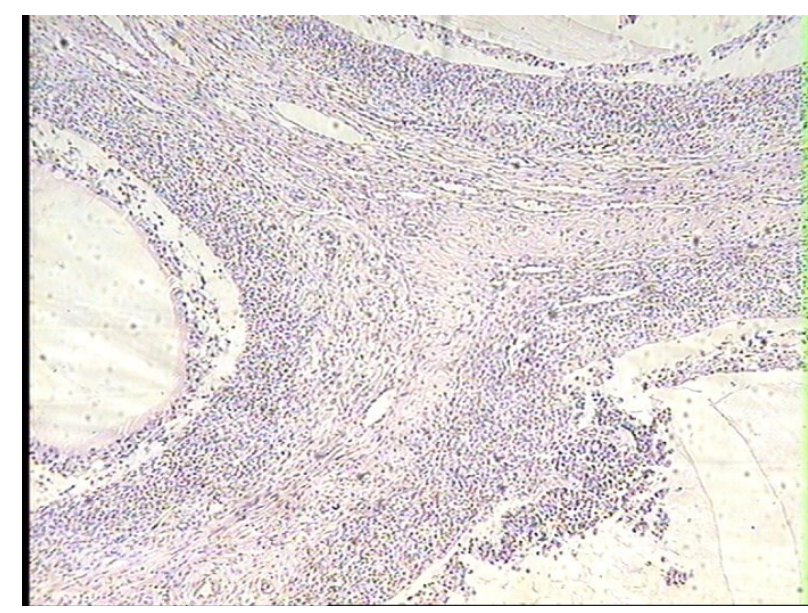

Fig. 9. Ovarian PEComa associated with cystic changes in ovarian follicles in a dairy cow with an adenomyotic uterus. HE, 10×

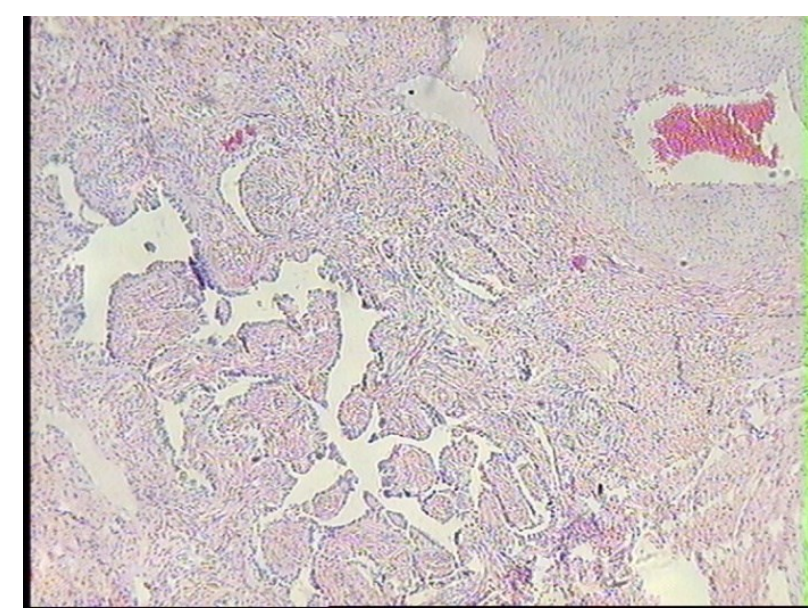

Fig. 10. Adenoma of rete ovarii cells in the ovary of a dairy cow with an adenomyotic uterus. HE, $10 \times$

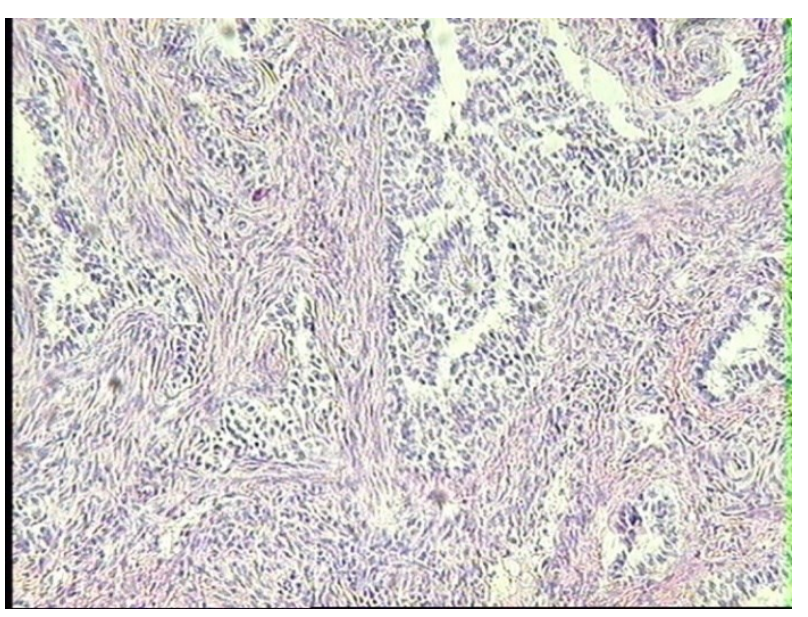

Fig. 11. Adenocarcinoma of rete ovarii cells in the ovary of a dairy cow with adenomyotic uterus. HE, $40 \times$

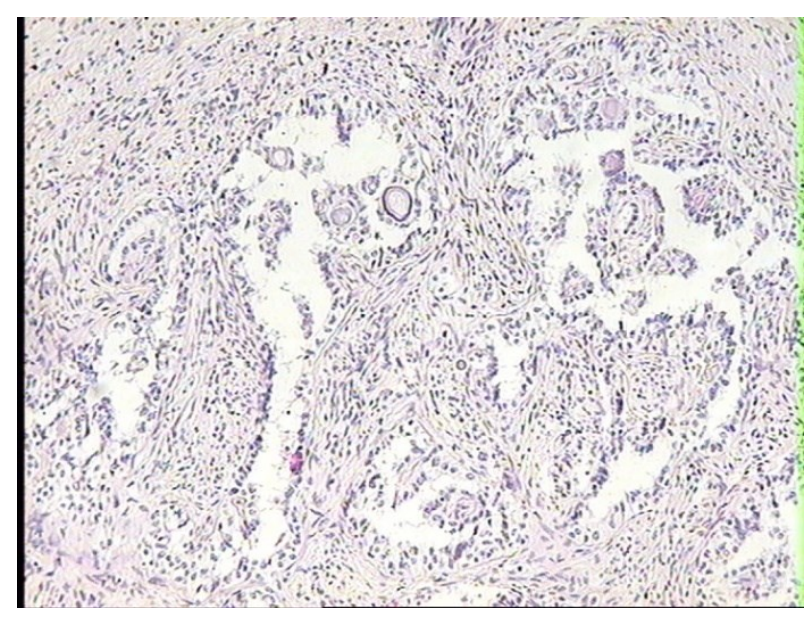

Fig. 12. The minute foci of granulosa cell tumour associated with adenoma of rete ovarii cells the ovary of a dairy cow with an adenomyotic uterus. HE, $20 \times$ 
The presented data may be valuable for the interpretation of the aetiology of infertility in dairy cows with clinically latent disease symptoms, or with disturbances in udder function. The evidence of the fertility problems in these cows is provided by the pathological lesions found in the ovarian follicles. It must be emphasised that the early phases of the described diseases are difficult and sometimes/impossible for veterinary practitioners to diagnose by using the methods of clinical examination favoured in living animals.

The primary endocrine hormones imbalance present in the examined dairy cows, due to which the uterine and mammary gland cell injury develop, may also cause complex ovarian cell metaplasia. It is attested to by various types of tumour growth in the same ovary. There were PEComa and fibroadenoma of rete ovarii, PEComa and haemangioma, and PEComa alone developing besides small foci of granulosa cell tumour. This complex neoplasia was found in single cases, but it can indicate that various types of ovarian cells are susceptible to broadly defined pathological hormonal stimulation. The observed differences in the degree and type of pathological lesions found in the investigated ovaries suggested great variability in the character of the hormones imbalance present in the sampled dairy cows' population. This hypothesis may merit consideration in any attempt to develop concentration standards for the specific ovarian hormones.

In conclusion, the results of this study make some progress in advancing veterinary knowledge of ovarian pathology processes. They may also be valuable in comparative researches as an animal model for the investigation of the pathological factors involved in ovarian cell neoplastic changes.

Conflict of Interest Statement: The author declares that there is no conflict of interests regarding the publication of this article.

Financial Disclosure Statement: Not applicable.

Animal Rights Statement: None required.

\section{References}

1. Byskov A.G.: Does rete ovarii act as a trigger for the onset of meiosis?. Nature, 1974, 252, 396-387.

2. Froio E., Piana S., Cavazza A., Valli R., Abrate M., Giardini G.: Multifocal PEComa (PEComatosis) of the female genital tract associated with endometriosis, diffuse adenomyosis, and endometrial atypical hyperplasia. Int J Surg Pathol 2008, 16, 443-446, doi: 10.1177/1066896908316067.

3. Jones T.C., Hunt R.D., King N.W.: Veterinary Pathology, $6^{\text {th }}$ ed. 1997, Lippincott Williams@ @ilkins, Philadelphia.

4. Katkiewicz M.: PEComa - the family of mesenchymal tumors Życie Wet 2017, 92, 369-370.

5. Katkiewicz M., Jurka P.: Changes in ovaries microscopic structure after estradiol administration in bitches. Życie Wet 2018, 92, $41-44$.

6. Katkiewicz M., Wierzchon M., Boryczko Z.: Uterus biopsy in milking cows used for detection of infertility. Weterynaria w Terenie, 2019, 2, 20-25.

7. Katkiewicz M., Witkowski M.: The histopathological changes in rete ovarii in cows with uterus adenomyosis and chronic mastits. Życie Wet 2014, 89, 214-219.

8. Katkiewicz M., Witkowski M.: Histopathological changes of rete ovarii in bitches with cystic endometrial hyperplasia/pyometra complex. Życie Wet 2015, 90, 595-599.

9. Keller L.S.F., Griffith J.W., Lang C.M.: Reproductive failure associated with cystic rete ovarii in guinea pigs. Vet Pathol 1987, $24,355-339$.

10. Khan M.S., Dodson A.R., Heatley M.K.: Ki-67, oestrogen receptor, and progesterone receptor proteins in the human rete ovarii and in endometriosis. J Clin Pathol 1999, 52, 517-520, doi:10.1136/jcp.52.7.517.

11. Maclachan N.J., Kennedy P.C.:Tumor of the genital system pp. 550-551 In: Meuten D.J.: Tumors in Domestic Animals, $4^{\text {th }}$ ed., 2002, Iowa State Press, Ames, Iowa.

12. Marr-Belvin A.K., Bailey C.C., Knight H.L., Klump S.A., Westmoreland S.V., Miller A.D.: Ovarian pathology in rhesus macaques: a 2 year retrospective. J Med Primatol 2010, 19, 170-176, doi:10.1111/j.1600-0684.2010.00409.x.

13. Musella A., De Felice F., Kyriacou A.K., BarlettaF., Di Matteo F.M.,Marchetti C., Izzo L., Monti M., Panici P.B., Redler A., D'Andrea V.: Perivascular epithelioid cell neoplasm (PEComa) of the uterus: A systematic review. Int J Surg 2015, 19, 1-5, doi: 10.1016/j.ijsu.2015.05.002.

14. Wenzel J.G., Odend'hal S.: The mammalian rete ovarii: a literature review. Cornell Vet 1985, 75, 411-425.

15. Westaby J., Magdy N., Fisher C., El-Bahravy M.: Primary Ovarian Malignant PEComa. A Case Report. Int J Gyn Pathol 2017, 36, 400-404.

16. WHO: WHO Classification of Tumours, $3^{\text {rd }}$ edition, volume 5: Pathology and Genetics of Tumours of Soft Tissue and Bone, edited by C.D.M. Fletcher, K.K. Unni, F. Martens. IARC Publication, Lyon 2002. 